\title{
Joint Beamforming Optimization and Power Control for Full-Duplex MIMO Two-way Relay Channel
}

\author{
Gan Zheng, Senior Member, IEEE
}

\begin{abstract}
In this paper we explore the use of full-duplex radio to improve the spectrum efficiency in a two-way relay channel where two sources exchange information through an multi-antenna relay, and all nodes work in the full-duplex mode. The full-duplex operation can reduce the overall communication to only one phase but suffers from the self-interference. Instead of purely suppressing the self-interference, we aim to maximize the end-to-end performance by jointly optimizing the beamforming matrix at the relay which uses the amplify-and-forward protocol as well as the power control at the sources. To be specific, we propose iterative algorithms and 1-D search to solve two problems: finding the achievable rate region and maximizing the sum rate. At each iteration, either the analytical solution or convex formulation is obtained. We compare the proposed fullduplex two-way relaying with the conventional half-duplex twoway relaying, a full-duplex one-way relaying and a performance upper bound. Numerical results show that the proposed fullduplex scheme significantly improves the achievable data rates over the conventional scheme.
\end{abstract}

Index Terms-Two-way relay channel, full-duplex radios, physical layer network coding, beamforming, optimization

\section{INTRODUCTION}

Cooperative communications via relaying is an effective measure to ensure reliability, provide high throughput and extend network coverage. It has been intensively studied in LTE-Advanced [1] and will continue to play an important role in the future fifth generation wireless networks. In the conventional two-hop one-way relay channel (OWRC), the communication takes two orthogonal phases, and suffers from the loss of spectral efficiency because of the inherent halfduplex (HD) constraint at the relay. Two-way relaying using the principle of physical layer network coding is proposed to recover the loss in OWRC by allowing the two sources to exchange information more efficiently [2][3][4]. In the first phase of the two-way relay channel (TWRC), both sources transmit signals to the relay. In the second phase, the relay does not separate signals but rather broadcasts the processed mixed signals to both sources. Each source can subtract its own message from the received signal then decodes the information from the other source. The benefit of the TWRC is that, using the same two communication phases as the OWRC, bidirectional communication is achieved.

However, note that the relay in the TWRC still operates in the HD mode thus two communication phases are needed. Motivated by this observation and thanks to the emerging

Gan Zheng is with School of Computer Science and Electronic Engineering, University of Essex, UK, E-mail: ganzheng@essex.ac.uk. He is also affiliated with Interdisciplinary Centre for Security, Reliability and Trust (SnT), University of Luxembourg, Luxembourg. full-duplex (FD) techniques, we aim to study the potential of the FD operation in the TWRC to enable simultaneous information exchange between the two sources, i.e., only one communication phase is required. In the proposed scheme, all nodes, including the two sources and the relay, work in the FD mode so they can transmit and receive signals simultaneously [5] [6]. However, the major challenge in the FD operation is that the power of the self-interference (SI) from the relay output could be over $100 \mathrm{~dB}$ higher than that of the signal received from distance sources and well exceeds the dynamic range of the analog-to-digital converter [7], [8], [9]. Therefore it is important that the SI is sufficiently mitigated. The SI cancellation can be broadly categorized as passive cancellation and active cancellation [10]. Passive suppression is to isolate the transmit and receive antennas using techniques such as directional antennas, absorptive shielding and cross-polarization [11]. Active suppression is to exploit a node's knowledge of its own transmit signal to cancel the SI, which typically includes analog cancellation, digital cancellation and spatial cancellation. Experimental results are reported in [12] that the SI can be cancelled to make FD wireless communication feasible in many cases. In the recent work [13] and [14], the promising results show that the SI can be suppressed to the noise level in both single-antenna and multi-antenna cases. Below we will provide a review on the application of the FD relaying to both the OWRC and TWRC.

\section{A. Literature on the FD relaying}

1) OWRC: The FD operation has attracted lots of research interest for relay assisted cooperative communication. It is shown in [7] that the FD relaying is feasible even in the presence of the SI and can offer higher capacity than the HD mode. Multiple-input multiple-output (MIMO) techniques provide an effective means to suppress the SI in the spatial domain [15]-[19]. The authors of [15] analyze a wide range of SI mitigation measures when the relay has multiple antennas, including natural isolation, time-domain cancellation and spatial domain suppression. The FD relay selection is studied in [16] for the amplify-and-forward (AF) cooperative networks. With multiple transmit or receive antennas at the full-duplex relay, precoding at the transmitter and decoding at the receiver can be jointly optimized to mitigate the SI effects. The joint precoding and decoding design for the FD relaying is studied in [15], where both the zero forcing (ZF) solutions and the minimum mean square error (MMSE) solutions are discussed. When only the relay has multiple antennas, a joint design of ZF precoding and decoding vectors is proposed in 
[17] to null out the SI at the relay. However, this design does not take into account the end-to-end (e2e) performance. A gradient projection method is proposed in [18], to optimize the precoding and decoding vectors considering the e2e performance. When all terminals have multiple antennas, the e2e performance is optimized in [19] where the closedform solution for precoding/decoding vectors design as well as diversity analysis are provided.

2) TWRC: In the early work of TWRC, the FD operation is often employed to investigate the capacity region from the information-theoretic viewpoint without considering the effects of the SI [20][21]. Only recently, the SI has been taken into account in the FD TWRC. In [22], the FD operation is introduced to the relay but two one-way relaying phases are required to achieve the two-way communication to avoid interference. A physical layer network coding FD TWRC is proposed in [23], where bit error rate is derived. The optimal relay selection scheme is proposed and analyzed in [24] for the FD TWRC using the AF protocol. Transmit power optimization among the source nodes and the relay node is studied in [25], again using the AF protocol. However, all existing works are restricted to the single transmit/receive antenna case at the relay thus the potential of using multiple antennas to suppress the SI and improve the e2e performance for the TWRC is not fully explored yet.

\section{B. Our work and contribution}

In this work, we study the potential of the MIMO FD operation in the TWRC where the relay has multiple transmit/receive antennas and employs the AF protocol and the principle of physical layer network coding. The two sources have single transmit/receive antenna. We jointly optimize the relay beamforming matrix and the power allocation at the sources to maximize the e2e performance. To be specific, we study two problems: one is to find the achievable rate region and the other is to maximize the sum rate of the FD TWRC. Our contributions are summarized as follows:

- We derive the signal model for the MIMO FD TWRC and propose to use the ZF constraint at the relay to greatly simplify the model and the problem formulations.

- We find the rate region by maximizing one source's rate with the constraint on the other source' minimum rate. We propose an iterative algorithm together with 1-D search to find the local optimum solution. At each iteration, we give analytical solutions for the transmit beamforming vector and the power control.

- We tackle the sum rate maximization problem by employing a similar iterative algorithm. At each iteration, we propose to use the DC (difference of convex functions) approach to optimize the transmit beamforming vector and we solve the power allocation analytically.

- We conduct intensive simulations to compare the proposed FD scheme with three benchmark schemes and clearly show its advantages of enlarging the rate region and improving the sum rate.

The rest of the paper is organized as follows. In Section II, we present the system model, the explicit signal model

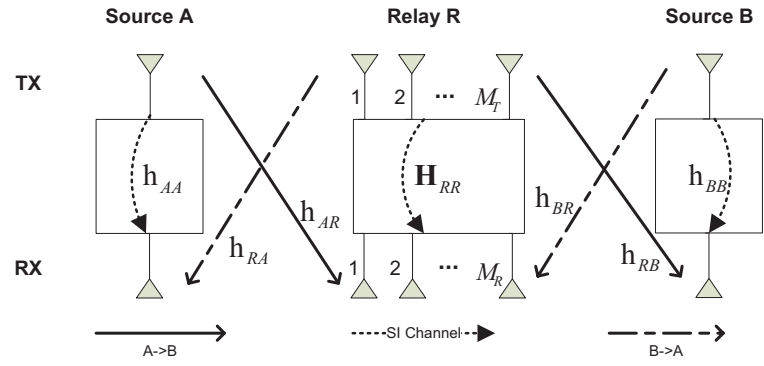

Fig. 1. Full-duplex TWRC with two sources and a MIMO relay. The dashed line denotes the residual self-interference.

and problem formulations. In Section III, we deal with the problem of finding the achievable rate region; in Section IV, we address the problem of maximizing the sum rate. Three benchmark schemes are introduced in Section V. Simulation results are presented in Section VI. Section VII concludes this paper and gives future directions.

Notation: The lowercase and uppercase boldface letters (e.g., $\mathbf{x}$ and $\mathbf{X}$ ) indicate column vectors and matrices, respectively. $\mathbf{X} \in \mathbb{C}^{M \times N}$ means a complex matrix $\mathbf{X}$ with dimension of $M \times N$. I is the identity matrix. We use $(\cdot)^{\dagger}$ to denote the conjugate transpose, $\operatorname{trace}(\cdot)$ is the trace operation, and $\|\cdot\|$ is the Frobenius norm. $|\cdot|$ represents the absolution value of a scalar. $\mathbf{X} \succeq \mathbf{0}$ denotes that the Hermitian matrix $\mathbf{X}$ is positive semidefinite. The expectation operator is denoted by $\mathcal{E}(\cdot)$. Define $\Pi_{\mathbf{X}}=\mathbf{X}\left(\mathbf{X}^{\dagger} \mathbf{X}\right)^{-1} \mathbf{X}^{\dagger}$ as the orthogonal projection onto the column space of $\mathbf{X}$; and $\Pi_{\mathbf{X}}^{\frac{1}{}}=\mathbf{I}-\Pi_{\mathbf{X}}$ as the orthogonal projection onto the orthogonal complement of the column space of $\mathbf{X}$.

\section{SYSTEM MODEL, SIGNAL MODEL AND PROBLEMS STATEMENT}

\section{A. System model}

Consider a three-node MIMO relay network consisting of two sources A and B who want to exchange information with the aid of a MIMO relay R, as depicted in Fig. 1. There is no direct link between the two sources because of deep fading or heavy shadowing, so their communication must rely on R. All nodes work in the FD mode. To enable the FD operation, each source is equipped with two groups of RF chains and corresponding antennas, i.e., one for transmitting and one for receiving signall]. We assume that each source has one transmit antenna and one receive antenna. We use $M_{T}$ and $M_{R}$ to denote the number of transmit and receive antennas at R, respectively. We assume $M_{T}>1$ or $M_{R}>1$ to help suppress the residual SI at $\mathrm{R}$ in the spatial domain. We use $\mathbf{h}_{X R} \in \mathbb{C}^{M_{R} \times 1}$ and $\mathbf{h}_{R X} \in \mathbb{C}^{M_{T} \times 1}$ to denote the directional channel vectors between the source node X's $(X \in\{A, B\})$ transmit antenna to R's receive antenna(s), and between R's transmit antenna(s) to X's receive antenna, respectively. In general, channel reciprocity does not hold, e.g., $\mathbf{h}_{X R} \neq \mathbf{h}_{R X}$, due to different transmit and receive antennas used. In addition, $h_{A A}, h_{B B}$ and $\mathbf{H}_{R R} \in \mathbb{C}^{M_{R} \times M_{T}}$

\footnotetext{
${ }^{1}$ It is also possible to realize the FD operation using a single antenna, see
} 
denote the residual SI channels after the SI cancellation scheme is applied at the corresponding nodes [16], [7]. The statistics of the residual SI channel are not well understood yet [26]. The experimental-based study in [27] has demonstrated that the amount of SI suppression achieved by a combined analog/digital cancellation technique is a complicated function of several system parameters. Therefore for simplicity, in this paper, we model each element of the residual SI channel as a Gaussian distributed random variable with zero mean and variance $\sigma_{X}^{2}, X \in\{A, B, R\}$. All channel links between two nodes are subject to independent flat fading. We assume that the channel coefficients between different nodes remain constant within a normalized time slot, but change independently from one slot to another according to a complex Gaussian distribution with zero mean and unit variance. The global channel state information (CSI) is available at the relay where the optimization will be performed. As will be seen in Fig. 11 this is critical for the relay to adapt its beamforming matrix and for the two sources to adjust their transmit power. The noise at the node $X$ 's $(X \in\{A, B, R\})$ receive antenna(s) is denoted as $n_{X}\left(\mathbf{n}_{X}\right)$ and modeled as complex additive white Gaussian noise with zero mean and unit variance.

Since each source has only a single transmit and receive antenna, a single data stream $S_{A}$ and $S_{B}$ are transmitted from $\mathrm{A}$ and $\mathrm{B}$, with the average power $p_{A}$ and $p_{B}$, respectively. To keep the complexity low, $\mathrm{R}$ employs linear processing, i.e., the AF protocol with an amplification matrix $\mathbf{W}$, to process the received signal. The node $\mathrm{X}$ has maximum transmit power constraint $P_{X}$. We will jointly optimize the transmit power of the two source nodes, $p_{A}$ and $p_{B}$ together with the amplification matrix at the relay to maximize the e2e system performance.

blueThe overhead due to the CSI acquisition at each node is analyzed as follows. The received CSI $\mathbf{h}_{A R}$ and $\mathbf{h}_{B R}$ at $R$ can be estimated by $A$ and $B$ each sending one pilot symbol separately. The SI channel $\mathbf{h}_{R R}$ can be estimated at $\mathrm{R}$ by itself sending an $M_{T}$-symbol pilot sequence. The SI channel $h_{A A}$ and $h_{B B}$ are estimated similarly and then sent back to R. Regarding the transmit CSI $\mathbf{h}_{R A}$ and $\mathbf{h}_{R B}$, $\mathrm{R}$ first broadcasts an $M_{T}$-symbol pilot sequence (this can be used for the estimation of $\mathbf{h}_{R R}$ simultaneously), then $\mathrm{A}$ and $\mathrm{B}$ feedback their estimation to $R$. In addition, after $R$ performs the optimization and obtains $\mathbf{W}, p_{A}$ and $p_{B}$, it transmits $\mathbf{h}_{R A}^{\dagger} \mathbf{W} \mathbf{h}_{A R}$ and $\mathbf{h}_{R B}^{\dagger} \mathbf{W} \mathbf{h}_{B R}$ to $\mathrm{A}$ and $\mathrm{B}$, respectively, such that they can cancel their previously sent symbols. Finally, $R$ informs $\mathrm{A}$ and $\mathrm{B}$ their transmit power $p_{A}$ and $p_{B}$, respectively.

\section{B. Signal model}

We assume that the processing delay at $R$ is given by bluea $\tau$-symbol duration, which refers to the required processing time in order to implement the FD operation [15]. $\tau$ typically takes integer values. The delay is short enough compared to a time slot which has a large number of data symbols, therefore its effect on the achievable rate is negligible. At the time (bluesymbol) instance $n$, the received signal $\mathbf{r}[n]$ and the transmit signal $\mathbf{x}_{R}[n]$ at $\mathrm{R}$ can be written as

$$
\mathbf{r}[n]=\mathbf{h}_{A R} S_{A}[n]+\mathbf{h}_{B R} S_{B}[n]+\mathbf{H}_{R R} \mathbf{x}_{R}[n]+\mathbf{n}_{R}[n],
$$

and

$$
\mathbf{x}_{R}[n]=\mathbf{W r}[n-\tau],
$$

respectively. Using (1) and (2) recursively, the overall relay output can be rewritten as

$$
\begin{aligned}
\mathbf{x}_{R}[n]= & \mathbf{W h}_{A R} S_{A}[n-\tau]+\mathbf{W} \mathbf{h}_{B R} S_{B}[n-\tau] \\
& +\mathbf{W H}_{R R} \mathbf{x}_{R}[n-\tau]+\mathbf{W} \mathbf{n}_{R}[n-\tau] \\
= & \mathbf{W} \sum_{j=0}^{\infty}\left(\mathbf{H}_{R R} \mathbf{W}\right)^{j}\left(\mathbf{h}_{A R} S_{A}[n-j \tau-\tau]\right. \\
& \left.+\mathbf{h}_{B R} S_{B}[n-j \tau-\tau]+\mathbf{n}_{R}[n-j \tau-\tau]\right),
\end{aligned}
$$

where $j$ denotes the index of the delayed symbols.

Its covariance matrix is given by

$$
\begin{aligned}
& \mathcal{E}\left[\mathbf{x}_{R} \mathbf{x}_{R}^{\dagger}\right]=P_{A} \mathbf{W} \sum_{j=0}^{\infty}\left(\mathbf{H}_{R R} \mathbf{W}\right)^{j} \mathbf{h}_{A R} \mathbf{h}_{A R}^{\dagger}\left(\left(\mathbf{H}_{R R} \mathbf{W}\right)^{j}\right)^{\dagger} \mathbf{W}^{\dagger} \\
& +P_{B} \mathbf{W} \sum_{j=0}^{\infty}\left(\mathbf{H}_{R R} \mathbf{W}\right)^{j} \mathbf{h}_{B R} \mathbf{h}_{B R}^{\dagger}\left(\left(\mathbf{H}_{R R} \mathbf{W}\right)^{j}\right)^{\dagger} \mathbf{W}^{\dagger} \\
& +\mathbf{W} \sum_{j=0}^{\infty}\left(\mathbf{H}_{R R} \mathbf{W} \mathbf{W}^{\dagger} \mathbf{H}_{R R}^{\dagger}\right)^{j} \mathbf{W}^{\dagger} \\
& =P_{A} \mathbf{W} \sum_{j=0}^{\infty}\left(\mathbf{H}_{R R} \mathbf{W}\right)^{j} \mathbf{h}_{A R} \mathbf{h}_{A R}^{\dagger}\left(\left(\mathbf{H}_{R R} \mathbf{W}\right)^{j}\right)^{\dagger} \mathbf{W}^{\dagger} \\
& +P_{B} \mathbf{W} \sum_{j=0}^{\infty}\left(\mathbf{H}_{R R} \mathbf{W}\right)^{j} \mathbf{h}_{B R} \mathbf{h}_{B R}^{\dagger}\left(\left(\mathbf{H}_{R R} \mathbf{W}\right)^{j}\right)^{\dagger} \mathbf{W}^{\dagger} \\
& +\mathbf{W}\left(\mathbf{I}-\mathbf{H}_{R R} \mathbf{W} \mathbf{W} \mathbf{H}_{R R}^{\dagger}\right)^{-1} \mathbf{W}^{\dagger} .
\end{aligned}
$$

Note that R's transmit signal covariance $\mathcal{E}\left[\mathbf{x}_{R} \mathbf{x}_{R}^{\dagger}\right]$, and in turn the transmit power and the SI power, are complicated functions of $\mathbf{W}$, which makes the optimization problems difficult. To simplify the signal model and make the optimization problems more tractable, we add the ZF constraint such that the optimization of $\mathbf{W}$ nulls out the residual SI from the relay output to relay input. To realize this, it is easy to check from (3) that the following condition is sufficient,

$$
\mathbf{W H}_{R R} \mathbf{W}=\mathbf{0} .
$$

Consequently, (3) becomes

$\mathbf{x}_{R}[n]=\mathbf{W}\left(\mathbf{h}_{A R} S_{A}[n-\tau]+\mathbf{h}_{B R} S_{B}[n-\tau]+\mathbf{n}_{R}[n-\tau]\right)$,

with the covariance matrix

$\mathcal{E}\left[\mathbf{x}_{R} \mathbf{x}_{R}^{\dagger}\right]=p_{A} \mathbf{W} \mathbf{h}_{A R} \mathbf{h}_{A R}^{\dagger} \mathbf{W}^{\dagger}+p_{B} \mathbf{W} \mathbf{h}_{B R} \mathbf{h}_{B R}^{\dagger} \mathbf{W}^{\dagger}+\mathbf{W} \mathbf{W}^{\dagger}$.

The relay output power is

$$
\begin{aligned}
p_{R} & =\operatorname{trace}\left(\mathcal{E}\left[\mathbf{x}_{R} \mathbf{x}_{R}^{\dagger}\right]\right) \\
& =p_{A}\left\|\mathbf{W} \mathbf{h}_{A R}\right\|^{2}+p_{B}\left\|\mathbf{W} \mathbf{h}_{B R}\right\|^{2}+\operatorname{trace}\left(\mathbf{W} \mathbf{W}^{\dagger}\right) .
\end{aligned}
$$

The received signal at the source A can be written as

$$
\begin{aligned}
r_{A}[n]= & \mathbf{h}_{R A}^{\dagger} \mathbf{x}_{R}[n]+h_{A A} S_{A}[n]+n_{A}[n] \\
= & \mathbf{h}_{R A}^{\dagger} \mathbf{W h}_{A R} S_{A}[n-\tau]+\mathbf{h}_{R A}^{\dagger} \mathbf{W h}_{B R} S_{B}[n-\tau] \\
& +\mathbf{h}_{R A}^{\dagger} \mathbf{W} \mathbf{n}_{R}[n]+h_{A A} S_{A}[n]+n_{A}[n] .
\end{aligned}
$$

After cancelling its own transmitted signal $S_{A}[n-\tau]$, it becomes 2

$$
\begin{aligned}
r_{A}[n]= & \mathbf{h}_{R A}^{\dagger} \mathbf{W} \mathbf{h}_{B R} S_{B}[n-\tau]+\mathbf{h}_{R A}^{\dagger} \mathbf{W} \mathbf{n}_{R}[n] \\
& +h_{A A} S_{A}[n]+n_{A}[n] .
\end{aligned}
$$

${ }^{2}$ Note that different from $S_{A}[n-\tau], S_{A}[n]$ can not be completely cancelled due to the simultaneous transmission, which is the main challenge of the FD radio. 
The received signal-to-interference-plus-noise ratio (SINR) at the source A, denoted as $\gamma_{A}$, is expressed as

$$
\gamma_{A}=\frac{p_{B}\left|\mathbf{h}_{R A}^{\dagger} \mathbf{W} \mathbf{h}_{B R}\right|^{2}}{\left\|\mathbf{h}_{R A}^{\dagger} \mathbf{W}\right\|^{2}+p_{A}\left|h_{A A}\right|^{2}+1} .
$$

Similarly, the received SINR $\gamma_{B}$ at the source B can be written as

$$
\gamma_{B}=\frac{p_{A}\left|\mathbf{h}_{R B}^{\dagger} \mathbf{W} \mathbf{h}_{A R}\right|^{2}}{\left\|\mathbf{h}_{R B}^{\dagger} \mathbf{W}\right\|^{2}+p_{B}\left|h_{B B}\right|^{2}+1} .
$$

The achievable rates are then given by $R_{A}=\log _{2}\left(1+\gamma_{A}\right)$ and $R_{B}=\log _{2}\left(1+\gamma_{B}\right)$, respectively.

\section{Problems Statement}

The conventional physical layer analog network coding scheme requires two phases for $\mathrm{A}$ and $\mathrm{B}$ to exchange information [3]. Thanks to the FD operation, the proposed scheme reduces the whole communication to only one phase thus substantially increases the spectrum efficiency. However, the FD operation also brings the SI to each node so they may not always use their maximum power because higher transmit power also increases the level of the residual SI, therefore each node needs to carefully choose its transmit power.

We are interested in two e2e objectives subject to each source's power constraints by optimizing the relay beamforming and power allocation at each source. The first one is to find the achievable rate region $\left(R_{A}, R_{B}\right)$. This can be achieved by maximizing source A's rate while varying the constraint on the minimum rate of source B's rate (or vice versa), i.e., solving the rate maximization problem $\mathbb{P}_{1}$ below:

$\mathbb{P}_{1}: \max _{\mathbf{W}, p_{A}, p_{B}, p_{R}} R_{A}$ s.t. $\quad R_{B} \geq r_{B}, p_{X} \leq P_{X}, X \in\{A, B, R\}$ where $r_{B}$ is the constraint on source B's rate. By enumerating $r_{B}$, we can find the boundary of the achievable rate region.

The second problem is to maximize the sum rate of the two sources. Mathematically, this problem is formulated as $\mathbb{P}_{2}$ below:

$\mathbb{P}_{2}: \max _{\mathbf{W}, p_{A}, p_{B}, p_{R}} R_{A}+R_{B}$ s.t. $p_{X} \leq P_{X}, X \in\{A, B, R\}$.

The next two sections will be devoted to solving $\mathbb{P}_{1}$ and $\mathbb{P}_{2}$, respectively.

\section{FINDING THE ACHIEVABLE RATE REGION}

In this section, we aim to optimize the relay beamforming matrix $\mathbf{W}$ and the sources' transmit power $\left(p_{A}, p_{B}\right)$ to find the achievable rate region. This can be achieved by solving $\mathbb{P}_{1}$. Using the monotonicity between the SINR and the rate, it can be expanded as

$$
\begin{aligned}
& \max _{\mathbf{W}, p_{A}, p_{B}} \frac{p_{B}\left|\mathbf{h}_{R A}^{\dagger} \mathbf{W} \mathbf{h}_{B R}\right|^{2}}{\left\|\mathbf{h}_{R A}^{\dagger} \mathbf{W}\right\|^{2}+p_{A}\left|h_{A A}\right|^{2}+1} \\
& \text { s.t. } \quad \frac{p_{A}\left|\mathbf{h}_{R B}^{\dagger} \mathbf{W} \mathbf{h}_{A R}\right|^{2}}{\left\|\mathbf{h}_{R B}^{\dagger} \mathbf{W}\right\|^{2}+p_{B}\left|h_{B B}\right|^{2}+1} \geq \Gamma_{B}, \\
& p_{A}\left\|\mathbf{W h}_{A R}\right\|^{2}+p_{B}\left\|\mathbf{W h}_{B R}\right\|^{2}+\operatorname{trace}\left(\mathbf{W} \mathbf{W}^{\dagger}\right) \leq P_{R}(15) \\
& \mathbf{W} \mathbf{H}_{R R} \mathbf{W}=\mathbf{0}, \\
& 0 \leq p_{A} \leq P_{A}, 0 \leq p_{B} \leq P_{B},
\end{aligned}
$$

where $\Gamma_{B} \triangleq 2^{r_{B}}-1$ is the equivalent SINR constraint for the source B. Observe that all terms in 13 are quadratic in $\mathbf{W}$ except the ZF constraint $\mathbf{W} \mathbf{H}_{R R} \mathbf{W}=\mathbf{0}$, which is difficult to handle. Considering the fact that each source only transmits a single data stream and the network coding principle encourages mixing rather than separating the data streams from different sources, we decompose $\mathbf{W}$ as $\mathbf{W}=\mathbf{w}_{t} \mathbf{w}_{r}^{\dagger}$, where $\mathbf{w}_{r}$ is the receive beamforming vector and $\mathbf{w}_{t}$ is the transmit beamforming vector at $\mathrm{R}$. Then the $\mathrm{ZF}$ condition is simplified to $\left(\mathbf{w}_{r}^{\dagger} \mathbf{H}_{R R} \mathbf{w}_{t}\right) \mathbf{W}=\mathbf{0}$ or equivalently $\mathbf{w}_{r}^{\dagger} \mathbf{H}_{R R} \mathbf{w}_{t}=0$ because in general $\mathbf{W} \neq \mathbf{0}$. Without loss of optimality, we further assume $\left\|\mathbf{w}_{r}\right\|=1$. As a result, the problem (13) can be simplified to

$$
\begin{aligned}
& \max _{\mathbf{w}_{r}, \mathbf{w}_{t}, p_{A}, p_{B}} \frac{p_{B}\left|\mathbf{h}_{R A}^{\dagger} \mathbf{w}_{t}\right|^{2}\left|\mathbf{w}_{r}^{\dagger} \mathbf{h}_{B R}\right|^{2}}{\left|\mathbf{h}_{R A}^{\dagger} \mathbf{w}_{t}\right|^{2}+p_{A}\left|h_{A A}\right|^{2}+1} \\
& \text { s.t. } \quad \frac{p_{A}\left|\mathbf{h}_{R B}^{\dagger} \mathbf{w}_{t}\right|^{2}\left|\mathbf{w}_{r}^{\dagger} \mathbf{h}_{A R}\right|^{2}}{\left|\mathbf{h}_{R B}^{\dagger} \mathbf{w}_{t}\right|^{2}+p_{B}\left|h_{B B}\right|^{2}+1} \geq \Gamma_{B} \text {, } \\
& p_{A}\left\|\mathbf{w}_{t}\right\|^{2}\left|\mathbf{w}_{r}^{\dagger} \mathbf{h}_{A R}\right|^{2}+p_{B}\left\|\mathbf{w}_{t}\right\|^{2}\left|\mathbf{w}_{r}^{\dagger} \mathbf{h}_{B R}\right|^{2} \\
& +\left\|\mathbf{w}_{t}\right\|^{2} \leq P_{R} \text {, } \\
& \mathbf{w}_{r}^{\dagger} \mathbf{H}_{R R} \mathbf{w}_{t}=0 \text {, } \\
& \left\|\mathbf{w}_{r}\right\|=1 \text {, } \\
& 0 \leq p_{A} \leq P_{A}, 0 \leq p_{B} \leq P_{B} \text {. }
\end{aligned}
$$

Note that in order to guarantee the feasibility of the $\mathrm{ZF}$ constraint $\mathbf{w}_{r}^{\dagger} \mathbf{H}_{R R} \mathbf{w}_{t}=0, \mathrm{R}$ only needs to have two or more either transmit or receive antennas but not necessarily both.

The problem (16) is still quite complicated as variables $\mathbf{w}_{t}$, $\mathbf{w}_{r}$ and $\left(p_{A}, p_{B}\right)$ are coupled. Our idea to tackle this difficulty is to use an alternating optimization approach, i.e., at each iteration, we optimize one variable while keeping the other fixed, together with 1-D search to find $\mathbf{w}_{r}$. Details are given below.

\section{A. Parameterization of the receive beamforming vector $\mathbf{w}_{r}$}

Observe that $\mathbf{w}_{r}$ is mainly involved in $\left|\mathbf{w}_{r}^{\dagger} \mathbf{h}_{B R}\right|^{2}$ and $\left|\mathbf{w}_{r}^{\dagger} \mathbf{h}_{A R}\right|^{2}$, so it has to balance the signals received from the two sources. According to the result in [28], $\mathbf{w}_{r}$ can be parameterized by $0 \leq \alpha \leq 1$ as below:

$$
\mathbf{w}_{r}=\alpha \frac{\Pi_{\mathbf{h}_{B R}} \mathbf{h}_{A R}}{\left\|\Pi_{\mathbf{h}_{B R}} \mathbf{h}_{A R}\right\|}+\sqrt{1-\alpha} \frac{\Pi_{\mathbf{h}_{B R}}^{\perp} \mathbf{h}_{A R}}{\left\|\Pi_{\mathbf{h}_{B R}}^{\perp} \mathbf{h}_{A R}\right\|} .
$$

We have to remark that 17 is not the complete characterization of $\mathbf{w}_{r}$ because it is also involved in the $\mathrm{ZF}$ constraint $\mathbf{w}_{r}^{\dagger} \mathbf{H}_{R R} \mathbf{w}_{t}=0$, but this parameterization makes the problem more tractable.

Given $\alpha$, we can optimize $\mathbf{w}_{t}$ and $\left(p_{A}, p_{B}\right)$ as will be introduced below. Then we perform 1-D search to find the optimal $\alpha^{*}$. We will focus on how to separately optimize $\mathbf{w}_{t}$ and $\left(p_{A}, p_{B}\right)$ in the following two subsections. For the optimization of each variable, we will derive analytical solutions without using iterative methods or numerical algorithms.

\section{B. Optimization of the transmit beamforming vector $\mathbf{w}_{t}$}

We first look into the optimization of $\mathbf{w}_{t}$ assuming $\mathbf{w}_{r}$ and $\left(p_{A}, p_{B}\right)$ are fixed. Based on the problem $[16$, we get the 
following formulation:

$$
\begin{aligned}
& \max _{\mathbf{w}_{t}} \frac{p_{B}\left|\mathbf{h}_{R A}^{\dagger} \mathbf{w}_{t}\right|^{2}\left|\mathbf{w}_{r}^{\dagger} \mathbf{h}_{B R}\right|^{2}}{\left|\mathbf{h}_{R A}^{\dagger} \mathbf{w}_{t}\right|^{2}+p_{A}\left|h_{A A}\right|^{2}+1} \\
& \text { s.t. } \quad \frac{p_{A}\left|\mathbf{h}_{R B}^{\dagger} \mathbf{w}_{t}\right|^{2}\left|\mathbf{w}_{r}^{\dagger} \mathbf{h}_{A R}\right|^{2}}{\left|\mathbf{h}_{R B}^{\dagger} \mathbf{w}_{t}\right|^{2}+p_{B}\left|h_{B B}\right|^{2}+1} \geq \Gamma_{B} \text {, } \\
& p_{A}\left\|\mathbf{w}_{t}\right\|^{2}\left|\mathbf{w}_{r}^{\dagger} \mathbf{h}_{A R}\right|^{2}+p_{B}\left\|\mathbf{w}_{t}\right\|^{2}\left|\mathbf{w}_{r}^{\dagger} \mathbf{h}_{B R}\right|^{2} \\
& +\left\|\mathbf{w}_{t}\right\|^{2} \leq P_{R} \\
& \mathbf{w}_{r}^{\dagger} \mathbf{H}_{R R} \mathbf{w}_{t}=0 \text {. }
\end{aligned}
$$

By separating the variable $\mathbf{w}_{t}$ and using monotonicity, (18) is simplified to

$$
\begin{array}{cl}
\max _{\mathbf{w}_{t}} & \left|\mathbf{h}_{R A}^{\dagger} \mathbf{w}_{t}\right|^{2} \\
\text { s.t. } & \left|\mathbf{h}_{R B}^{\dagger} \mathbf{w}_{t}\right|^{2} \geq \frac{\Gamma_{B} p_{B}\left|h_{B B}\right|^{2}+1}{p_{A}\left|\mathbf{w}_{r}^{\dagger} \mathbf{h}_{A R}\right|^{2}-\Gamma_{B}} \triangleq \bar{\Gamma}_{B}, \\
& \left\|\mathbf{w}_{t}\right\|^{2} \leq \frac{P_{R}}{\left(p_{A}\left|\mathbf{w}_{r}^{\dagger} \mathbf{h}_{A R}\right|^{2}+p_{B}\left|\mathbf{w}_{r}^{\dagger} \mathbf{h}_{B R}\right|^{2}+1\right)} \triangleq \bar{P}, \\
& \mathbf{w}_{r}^{\dagger} \mathbf{H}_{R R} \mathbf{w}_{t}=0 .
\end{array}
$$

The problem 19) is not convex but it is a quadratic problem in $\mathbf{w}_{t}$. By defining $\mathbf{W}_{t}=\mathbf{w}_{t} \mathbf{w}_{t}^{\dagger}$ and using semidefinite programming relaxation, 19) will become a convex problem in $\mathbf{W}_{t}$, from which the optimal $\mathbf{w}_{t}$ can be found from matrix decomposition. Interested readers are referred to [29] for details. However, the special structure of the problem 19. allows us to derive the analytical solution in the following steps.

1) If $\bar{\Gamma}_{B}<0$ or $p_{A}\left|\mathbf{w}_{r}^{\dagger} \mathbf{h}_{A R}\right|^{2}<\Gamma_{B}$, the problem is infeasible; otherwise continue.

2) Define the null space of the vector $\mathbf{w}_{r}^{\dagger} \mathbf{H}_{R R}$ as $\mathbf{N}_{t} \in$ $\mathbb{C}^{M_{t} \times\left(M_{t}-1\right)}$, i.e., $\mathbf{w}_{r}^{\dagger} \mathbf{H}_{R R} \mathbf{N}_{t}=\mathbf{0}$. Introduce a new variable $\mathbf{v} \in \mathbb{C}^{\left(M_{t}-1\right) \times 1}$ and express $\mathbf{w}_{t}=\mathbf{N}_{t} \mathbf{v}$, then we can remove the $\mathrm{ZF}$ constraint in 19 , and obtain the following equivalent problem:

$$
\begin{array}{cl}
\max _{\mathbf{v}} & \left|\mathbf{h}_{R A}^{\dagger} \mathbf{N}_{t} \mathbf{v}\right|^{2} \\
\text { s.t. } & \left|\mathbf{h}_{R B}^{\dagger} \mathbf{N}_{t} \mathbf{v}\right|^{2} \geq \bar{\Gamma}_{B} \\
& \|\mathbf{v}\|^{2} \leq \bar{P},
\end{array}
$$

where we have used the property that $\mathbf{N}_{t}^{\dagger} \mathbf{N}_{t}=\mathbf{I}$. If $\bar{P}\left\|\mathbf{h}_{R B}^{\dagger} \mathbf{N}_{t}\right\|^{2}<\bar{\Gamma}_{B}$, the problem is infeasible; otherwise continue.

3) In this step we aim to find the closed-form solution for 20. . We first solve it without the constraint 21. It can be seen that the last power constraint should always be satisfied with equality, and the optimal solution is given by $\mathbf{v}^{*}=\sqrt{\bar{P}} \frac{\mathbf{N}_{t}^{\dagger} \mathbf{h}_{R A}}{\left\|\mathbf{N}_{t}^{\dagger} \mathbf{h}_{R A}\right\|}$. If it also satisfies the constraint 21], then it is the optimal solution; otherwise continue.

4) It this step, we know that both constraints in 20 should be active, so we reach the problem below:

$$
\begin{array}{cl}
\max _{\mathbf{v}} & \left|\mathbf{h}_{R A}^{\dagger} \mathbf{N}_{t} \mathbf{v}\right|^{2} \\
\text { s.t. } & \left|\mathbf{h}_{R B}^{\dagger} \mathbf{N}_{t} \mathbf{v}\right|^{2}=\bar{\Gamma}_{B} \\
& \|\mathbf{v}\|^{2}=\bar{P} .
\end{array}
$$

If we define $\mathbf{d}_{2} \triangleq \frac{\mathbf{N}_{t}^{\dagger} \mathbf{h}_{R A}}{\mathbf{N}_{t}^{\dagger} \mathbf{h}_{R A}}, \mathbf{d}_{1} \triangleq \frac{\mathbf{N}_{t}^{\dagger} \mathbf{h}_{R B}}{\mathbf{N}_{t}^{\dagger} \mathbf{h}_{R B}}, \phi \in(-\pi, \pi]$ be the argument of $\mathbf{d}_{2}^{\dagger} \mathbf{d}_{1}, r \triangleq\left|\mathbf{d}_{2}^{\dagger} \mathbf{d}_{1}\right|, q \triangleq \frac{\bar{\Gamma}_{B}}{\bar{P}\left\|\mathbf{N}_{t}^{\dagger} \mathbf{h}_{R B}\right\|^{2}}$ and $\mathbf{z} \triangleq \frac{\mathbf{v}}{\|\mathbf{v}\|}$, then we have the following formulation:

$$
\begin{array}{cl}
\max _{\mathbf{z}} & \mathbf{z}^{\dagger} \mathbf{d}_{2} \mathbf{d}_{2}^{\dagger} \mathbf{z}, \\
\text { s.t. } & \mathbf{z}^{\dagger} \mathbf{d}_{1} \mathbf{d}_{1}^{\dagger} \mathbf{z}=q, \quad\|\mathbf{z}\|=1 .
\end{array}
$$

The optimal solution $\mathbf{z}^{*}$ follows from Lemma 2 in [30] and is given below

$$
\mathbf{z}^{*}=\left(r \frac{1-q}{1-r^{2}}-\sqrt{q}\right) e^{j(\pi-\phi)} \mathbf{d}_{1}+\sqrt{\frac{1-q}{1-r^{2}}} \mathbf{d}_{2} .
$$

Once we obtain $\mathbf{z}^{*}$, the optimal transmit beamforming vector is given by

$$
\mathbf{w}_{t}^{*}=\mathbf{N}_{t} \mathbf{v}^{*}=\sqrt{\bar{P}} \mathbf{N}_{t} \mathbf{z}^{*}
$$

After obtaining the optimal $\mathbf{w}_{t}^{*}$ using the above procedures, we can move on to find the optimal power allocation at the sources.

\section{Optimization of the source power $\left(p_{A}, p_{B}\right)$}

Because of the FD operation at the sources and the fact that each source has a single transmit and receive antenna, they cannot suppress the residual SI in the spatial domain therefore cannot always use the full power. In contrast, $R$ has at least two transmit or receive antennas, so it can complete eliminate the SI and transmit using full power $P_{R}$. Here we aim to find the optimal power allocation $\left(p_{A}, p_{B}\right)$ at $\mathrm{A}$ and $\mathrm{B}$ assuming both $\mathbf{w}_{t}$ and $\mathbf{w}_{r}$ are fixed.

For convenience, define $C_{A t} \triangleq\left|\mathbf{h}_{R A}^{\dagger} \mathbf{w}_{t}\right|^{2}, C_{r B} \triangleq$ $\left|\mathbf{w}_{r}^{\dagger} \mathbf{h}_{B R}\right|^{2}, C_{B t} \triangleq\left|\mathbf{h}_{R B}^{\dagger} \mathbf{w}_{t}\right|^{2}, C_{r A} \triangleq\left|\mathbf{w}_{r}^{\dagger} \mathbf{h}_{A R}\right|^{2}$, then (16) becomes

$$
\begin{aligned}
\max _{p_{A}, p_{B}} & \frac{p_{B} C_{A t} C_{r B}}{C_{A t}+p_{A}\left|h_{A A}\right|^{2}+1} \\
\text { s.t. } & \frac{p_{A} C_{B t} C_{r A}}{C_{B t}+p_{B}\left|h_{B B}\right|^{2}+1} \geq \Gamma_{B} \\
& p_{A}\left\|\mathbf{w}_{t}\right\|^{2} C_{r A}+p_{B}\left\|\mathbf{w}_{t}\right\|^{2} C_{r B}+\left\|\mathbf{w}_{t}\right\|^{2} \leq P_{R}, \\
& 0 \leq p_{A} \leq P_{A}, 0 \leq p_{B} \leq P_{B} .
\end{aligned}
$$

The problem (26) is a linear-fractional programming problem, and can be converted to a linear programming problem [31, p. 151]. Again, thanks to its special structure, we can derive its analytical solutions below step by step.

1) First we check whether the constraint 27) is feasible. If $P_{A} C_{B t} C_{r A} \leq \Gamma_{B}$, then the problem is infeasible; otherwise, continue.

2) Next we solve (26) by ignoring the constraint (28). It is easy to check that at the optimum, at least one source should achieve its maximum power. The power allocation depends on two cases:
a) If $P_{A} \geq \frac{\Gamma_{B}\left(C_{B t}\left\|\mathbf{w}_{r}\right\|^{2}+P_{B}\left|h_{B B}\right|^{2}+1\right)}{C_{B t} C_{r A}}$, then $p_{B}=$ $P_{B}, p_{A}=\frac{\Gamma_{B}\left(C_{B t}\left\|\mathbf{w}_{r}\right\|^{2}+P_{B}\left|h_{B B}\right|^{2}+1\right)}{C_{B t} C_{r A}{ }_{p_{A} C_{B t} C_{r A}}}$; otherwise,
b) $p_{A}=P_{A}, p_{B}=\min \left(P_{B}, \frac{\frac{p_{A} C_{B t} C_{r A}}{\Gamma_{B}}-1-C_{B t}}{\left|h_{B B}\right|^{2}}\right)$.

3) We check whether the above obtained solution satisfies the constraint (27). If it does, then it is the optimal 
solution. Otherwise the constraint (27) should be met with equality.

4) The optimal power allocation is determined by the equation set below,

$$
\left\{\begin{array}{l}
p_{A} C_{B t} C_{r A}=\Gamma_{B}\left(C_{B t}+p_{B}\left|h_{B B}\right|^{2}+1\right), \\
p_{A}\left\|\mathbf{w}_{t}\right\|^{2} C_{r A}+p_{B}\left\|\mathbf{w}_{t}\right\|^{2} C_{r B}+\left\|\mathbf{w}_{t}\right\|^{2}=P_{R}
\end{array}\right.
$$

and the solution is given by

$$
\left\{\begin{array}{l}
p_{A}=\frac{\Gamma_{B}\left|h_{B B}\right|^{2}}{C_{B t}+C_{r A}} p_{B}+\frac{\Gamma_{B}\left(C_{B t}+1\right)}{C_{B t}+C_{r A}} \\
p_{B}=\frac{\frac{P_{R}}{\left\|\mathrm{w}_{t}\right\|^{2}}-1-\frac{\Gamma_{B} C_{r A}\left(C_{B t}+1\right)}{C_{B t} C_{r A}}}{\frac{\Gamma_{B} C_{r A}\left|h_{B B}\right|^{2}}{C_{B t} C_{r A}}+C_{r B}} .
\end{array}\right.
$$

\section{The overall algorithm}

Given an $\alpha$ or $\mathbf{w}_{r}$, we can iteratively optimize $\mathbf{w}_{t}$ and $\left(p_{A}, p_{B}\right)$ as above until convergence. The value of the objective function monotonically increases as the iteration goes thus converges to a local optimum. We can then conduct 1-D search over $0 \leq \alpha \leq 1$ to find the optimal $\alpha^{*}$ or $\mathbf{w}_{r}$. By enumerating source B's requirement $r_{B}$, we can numerically find the boundary of the achievable rate region.

About the complexity, we remark that at each iteration, the solutions of $\mathbf{w}_{t}$ and $\left(p_{A}, p_{B}\right)$ are given in simple closed-forms, so the associated complexity is low.

\section{MAXimizing The Sum Rate}

In this section, we aim to maximize the sum rate of the proposed FD TWRC, i.e., to solve $\mathbb{P}_{2}$, which is rewritten below,

$$
\begin{aligned}
\max _{\mathbf{w}_{t}, \mathbf{w}_{r} p_{A}, p_{B}} & \log _{2}\left(1+\frac{p_{B} C_{r B}\left|\mathbf{h}_{R A}^{\dagger} \mathbf{w}_{t}\right|^{2}}{\left|\mathbf{h}_{R A}^{\dagger} \mathbf{w}_{t}\right|^{2}+p_{A}\left|h_{A A}\right|^{2}+1}\right)(31) \\
& +\log _{2}\left(1+\frac{p_{A} C_{r A}\left|\mathbf{h}_{R B}^{\dagger} \mathbf{w}_{t}\right|^{2}}{\left|\mathbf{h}_{R B}^{\dagger} \mathbf{w}_{t}\right|^{2}+p_{B}\left|h_{B B}\right|^{2}+1}\right) \\
\text { s.t. } & \left\|\mathbf{w}_{t}\right\|^{2} \leq \frac{P_{R}}{p_{A} C_{r A}+p_{B} C_{r B}+1}, \\
& \mathbf{w}_{r}^{\dagger} \mathbf{H}_{R R} \mathbf{w}_{t}=0 .
\end{aligned}
$$

We will use the same characterization of (17) to find the optimal $\mathbf{w}_{r}$ via $1-\mathrm{D}$ search. We then concentrate on alternatingly optimizing the transmit beamforming vector $\mathbf{w}_{t}$ and the power allocation $\left(p_{A}, p_{B}\right)$.

\section{A. Optimization of the transmit beamforming vector $\mathbf{w}_{t}$}

We first study how to optimize $\mathbf{w}_{t}$ given $\mathbf{w}_{r}$ and $\left(p_{A}, p_{B}\right)$. For convenience, we define a semidefinite matrix $\mathbf{W}_{t}=$ $\mathbf{w}_{t} \mathbf{w}_{t}^{\dagger}$. Then the problem (31) becomes

$$
\begin{array}{cl}
\max _{\mathbf{W}_{t} \succeq \mathbf{0}} & F\left(\mathbf{W}_{t}\right) \\
\text { s.t. } & \operatorname{trace}(\mathbf{W}) \leq \frac{P_{R}}{p_{A} C_{r A}+p_{B} C_{r B}+1}, \\
& \operatorname{trace}\left(\mathbf{W}_{t} \mathbf{H}_{R R}^{\dagger} \mathbf{W}_{r} \mathbf{W}_{r}^{\dagger} \mathbf{H}_{R R}\right)=0, \\
& \operatorname{rank}\left(\mathbf{W}_{t}\right)=1,
\end{array}
$$

where $F\left(\mathbf{W}_{t}\right) \triangleq \log _{2}\left(1+\frac{p_{B} C_{r B} \operatorname{trace}\left(\mathbf{W}_{t} \mathbf{h}_{R A} \mathbf{h}_{R A}^{\dagger}\right)}{\operatorname{trace}\left(\mathbf{W}_{t} \mathbf{h}_{R A} \mathbf{h}_{R A}^{\dagger}\right)+p_{A}\left|h_{A A}\right|^{2}+1}\right)+$ $\log _{2}\left(1+\frac{p_{A} C_{r A} \operatorname{trace}\left(\mathbf{W}_{t} \mathbf{h}_{R B} \mathbf{h}_{R B}^{\dagger}\right)}{\operatorname{trace}\left(\mathbf{W}_{t} \mathbf{h}_{R B} \mathbf{h}_{R B}^{\dagger}\right)+p_{B}\left|h_{B B}\right|^{2}+1}\right)$. Clearly $F\left(\mathbf{W}_{t}\right)$ is not a concave function thus (32) is a cumbersome optimization problem. To tackle it, we propose to use the DC programming [32] to find a local optimum point. To this end, we express $F\left(\mathbf{W}_{t}\right)$, as a difference of two concave functions $f\left(\mathbf{W}_{t}\right)$ and $g\left(\mathbf{W}_{t}\right)$, i.e.,

$$
\begin{aligned}
& F\left(\mathbf{W}_{t}\right) \\
& \left.=\log _{2}\left(\left(p_{B} C_{r B}+1\right) \operatorname{trace}\left(\mathbf{W}_{t} \mathbf{h}_{R A} \mathbf{h}_{R A}^{\dagger}\right)+p_{A}\left|h_{A A}\right|^{2}+1\right)\right) \\
& -\log _{2}\left(\operatorname{trace}\left(\mathbf{W}_{t} \mathbf{h}_{R A} \mathbf{h}_{R A}^{\dagger}\right)+p_{A}\left|h_{A A}\right|^{2}+1\right) \\
& \left.+\log _{2}\left(\left(p_{A} C_{r A}+1\right) \operatorname{trace}\left(\mathbf{W}_{t} \mathbf{h}_{R B} \mathbf{h}_{R B}^{\dagger}\right)+p_{B}\left|h_{B B}\right|^{2}+1\right)\right) \\
& -\log _{2}\left(\operatorname{trace}\left(\mathbf{W}_{t} \mathbf{h}_{R B} \mathbf{h}_{R B}^{\dagger}\right)+p_{B}\left|h_{B B}\right|^{2}+1\right) \\
& \triangleq f\left(\mathbf{W}_{t}\right)-g\left(\mathbf{W}_{t}\right)
\end{aligned}
$$

where

$$
\begin{aligned}
f\left(\mathbf{W}_{t}\right) & \left.\triangleq \log _{2}\left(\left(p_{B} C_{r B}+1\right) \operatorname{trace}\left(\mathbf{W}_{t} \mathbf{h}_{R A} \mathbf{h}_{R A}^{\dagger}\right)+p_{A}\left|h_{A A}\right|^{2}+1\right)\right) \\
& \left.+\log _{2}\left(\left(p_{A} C_{r A}+1\right) \operatorname{trace}\left(\mathbf{W}_{t} \mathbf{h}_{R B} \mathbf{h}_{R B}^{\dagger}\right)+p_{B}\left|h_{B B}\right|^{2}+1\right)\right), \\
g\left(\mathbf{W}_{t}\right) & \triangleq \log _{2}\left(\operatorname{trace}\left(\mathbf{W}_{t} \mathbf{h}_{R A} \mathbf{h}_{R A}^{\dagger}\right)+p_{A}\left|h_{A A}\right|^{2}+1\right) \\
& +\log _{2}\left(\operatorname{trace}\left(\mathbf{W}_{t} \mathbf{h}_{R B} \mathbf{h}_{R B}^{\dagger}\right)+p_{B}\left|h_{B B}\right|^{2}+1\right) .
\end{aligned}
$$

$f\left(\mathbf{W}_{t}\right)$ is a concave function while $g\left(\mathbf{W}_{t}\right)$ is a convex function. The main idea is to approximate $g\left(\mathbf{W}_{t}\right)$ by a linear function. The linearization (first-order approximation) of $g\left(\mathbf{W}_{t}\right)$ around the point $\mathbf{W}_{t, k}$ is given by

$$
\begin{aligned}
& g_{L}\left(\mathbf{W}_{t} ; \mathbf{W}_{t, k}\right)=\frac{1}{\ln (2)} \frac{\operatorname{trace}\left(\left(\mathbf{W}_{t}-\mathbf{W}_{t, k}\right) \mathbf{h}_{R A} \mathbf{h}_{R A}^{\dagger}\right)}{\operatorname{trace}\left(\mathbf{W}_{t, k} \mathbf{h}_{R A} \mathbf{h}_{R A}^{\dagger}\right)+p_{A}\left|h_{A A}\right|^{2}+1} \\
& +\frac{1}{\ln (2)} \frac{\operatorname{trace}\left(\left(\mathbf{W}_{t}-\mathbf{W}_{t, k}\right) \mathbf{h}_{R B} \mathbf{h}_{R B}^{\dagger}\right)}{\operatorname{trace}\left(\mathbf{W}_{t, k} \mathbf{h}_{R B} \mathbf{h}_{R B}^{\dagger}\right)+p_{B}\left|h_{B B}\right|^{2}+1} \\
& +\log _{2}\left(\operatorname{trace}\left(\mathbf{W}_{t, k} \mathbf{h}_{R A} \mathbf{h}_{R A}^{\dagger}\right)+p_{A}\left|h_{A A}\right|^{2}+1\right) \\
& +\log _{2}\left(\operatorname{trace}\left(\mathbf{W}_{t, k} \mathbf{h}_{R A} \mathbf{h}_{R A}^{\dagger}\right)+p_{A}\left|h_{A A}\right|^{2}+1\right) .
\end{aligned}
$$

Then the DC programming is applied to sequentially solve the following convex problem,

$$
\begin{aligned}
\mathbf{W}_{t, k+1}= & \arg \max _{\mathbf{W}_{t}} f\left(\mathbf{W}_{t}\right)-g_{L}\left(\mathbf{W}_{t} ; \mathbf{W}_{t, k}\right) \\
& \text { s.t. } \operatorname{trace}\left(\mathbf{W}_{t}\right)=\frac{P_{R}}{p_{A} C_{r A}+p_{B} C_{r B}+1}, \\
& \operatorname{trace}\left(\mathbf{W}_{t} \mathbf{H}_{R R}^{\dagger} \mathbf{W}_{r} \mathbf{W}_{r}^{\dagger} \mathbf{H}_{R R}\right)=0 .
\end{aligned}
$$

To summarize, the problem (32) can be solved by i) choosing an initial point $\mathbf{W}_{t}$; and ii) for $k=0,1, \cdots$, solving 35 until the termination condition is met. Notice that in 35 we have ignored the rank-1 constraint on $\mathbf{W}_{t}$. This constraint is guaranteed to be satisfied by the results in [33, Theorem 2] when $M_{t}>2$, therefore the decomposition of $\mathbf{W}_{t}$ leads to the optimal solution $\mathrm{w}_{t}^{*}$ for 31. When $M_{t}=2$, the $\mathrm{ZF}$ constraint in the problem (31) can determine the direction of $\mathbf{w}_{t}$, i.e., $\mathbf{w}_{t}=\sqrt{p_{t}} \mathbb{N}_{t}$ where $p_{t}$ is the transmit power and $\mathbb{N}_{t} \in \mathbb{C}^{2 \times 1}$ represents the null space of $\mathbf{w}_{r}^{\dagger} \mathbf{H}_{R R}$. Therefore the optimization of $\mathbf{w}_{t}$ reduces to optimizing a scalar variable $p_{t}$, which can be found by checking the stationary points of the objective function in 31 and the boundary point without using the DC programming. The same applies to the special case of $M_{t}=1$. 


\section{B. Optimization of source power $\left(p_{A}, p_{B}\right)$}

With $\mathbf{w}_{t}$ and $\mathbf{w}_{r}$ fixed, the sum rate maximization problem (31) about power allocation can be written as

$$
\begin{array}{cl}
\max _{p_{A}, p_{B}} & \log _{2}\left(1+\frac{p_{B} C_{A t} C_{r B}}{C_{A t}+p_{A}\left|h_{A A}\right|^{2}+1}\right) \\
& +\log _{2}\left(1+\frac{p_{A} C_{B t} C_{r A}}{C_{B t}+p_{B}\left|h_{B B}\right|^{2}+1}\right) \\
\text { s.t. } & p_{A} C_{r A}+p_{B} C_{r B}+1 \leq \frac{P_{R}}{\left\|\mathbf{w}_{t}\right\|^{2}}, \\
& 0 \leq p_{A} \leq P_{A}, 0 \leq p_{B} \leq P_{B} .
\end{array}
$$

Note that when the first relay power constraint (37) is not tight, the problem is the same as the conventional power allocation among two interference links to maximize the sum rate, and the optimal power solution is known to be binary [34], i.e., the optimal power allocation $\left(p_{A}^{*}, p_{B}^{*}\right)$ should satisfy

$$
\left(p_{A}^{*}, p_{B}^{*}\right) \in\left\{\left(0, P_{B}\right),\left(P_{A}, 0\right),\left(P_{A}, P_{B}\right)\right\} .
$$

Next we only focus on the case in which the constraint 37 is active, i.e., $p_{A} C_{r A}+p_{B} C_{r B}+1=\frac{P_{R}}{\left\|\mathbf{w}_{t}\right\|^{2}}$. We then have

$$
p_{A}=\frac{\frac{P_{R}}{\left\|\mathbf{w}_{t}\right\|^{2}-1}-p_{B} C_{r B}}{C_{r A}} .
$$

Because $0 \leq p_{A} \leq P_{A}$, we can obtain the feasible range $\left[p_{B}^{\min }, p_{B}^{\max }\right]$ for $p_{B}$ :

$$
\begin{aligned}
& p_{B}^{\min }=\max \left(0, \frac{P_{R}\left\|\mathbf{w}_{t}\right\|^{2}-1-C_{r A} P_{A}}{C_{r B}}\right), \\
& p_{B}^{\max }=\min \left(P_{B}, \frac{P_{R}\left\|\mathbf{w}_{t}\right\|^{2}-1}{C_{r B}}\right) .
\end{aligned}
$$

The objective function of (36) then becomes a function of $p_{B}$ only, i.e.,

$$
\begin{aligned}
y\left(p_{B}\right)= & \log _{2}\left(C_{A t}+p_{A}\left|h_{A A}\right|^{2}+1+p_{B} C_{A t} C_{r B}\right) \\
& -\log _{2}\left(C_{A t}+p_{A}\left|h_{A A}\right|^{2}+1\right) \\
& +\log _{2}\left(C_{B t}+p_{B}\left|h_{B B}\right|^{2}+1+p_{A} C_{B t} C_{r A}\right) \\
& -\log _{2}\left(C_{B t}+p_{B}\left|h_{B B}\right|^{2}+1\right),
\end{aligned}
$$

and (36) reduces to a one-variable optimization, i.e.,

$$
\max _{p_{B}} y\left(p_{B}\right) \quad \text { s.t. } p_{B}^{\min } \leq p_{B} \leq p_{B}^{\max } .
$$

Setting $\frac{\partial y\left(p_{B}\right)}{\partial p_{B}}=0$ leads to

$$
\begin{aligned}
& \frac{C_{A t} C_{r B}-\frac{C_{r B}}{C_{r A}}\left|h_{A A}\right|^{2}}{C_{A t}+\frac{\frac{P_{R}}{\left\|\mathrm{w}_{t}\right\|^{2}-1}\left|h_{A A}\right|^{2}}{C_{r A}}+1+p_{B}\left(C_{A t} C_{r B}-\frac{C_{r B}}{C_{r A}}\left|h_{A A}\right|^{2}\right)} \\
& +\frac{\frac{C_{r B}}{C_{r A}}\left|h_{A A}\right|^{2}}{C_{A t}+\frac{\frac{P_{R}}{\left\|\mathbf{w}_{t}\right\|^{2}-1}}{C_{r A}}\left|h_{A A}\right|^{2}+1-p_{B} \frac{C_{r B}}{C_{r A}}\left|h_{A A}\right|^{2}} \\
& +\frac{\left|h_{B B}\right|^{2}-\frac{C_{r B}}{C_{r A}} C_{B t} C_{r A}}{C_{B t}+\frac{\frac{P_{R}}{\left\|\mathbf{w}_{t}\right\|^{2}-1}}{C_{r A}} C_{B t} C_{r A}+1+p_{B}\left(\left|h_{B B}\right|^{2}-\frac{C_{r B}}{C_{r A}} C_{B t} C_{r A}\right)} \\
& -\frac{\left|h_{B B}\right|^{2}}{C_{B t}+p_{B}\left|h_{B B}\right|^{2}+1}=0 .
\end{aligned}
$$

This in turn becomes a cubic (3-rd order) equation and all roots can be found analytically. Suppose that the set of all
$\mathbb{P}_{1}$ and $\mathbb{P}_{2}$ can be solved to find the achievable rate region and the maximum sum rate, respectively. Compared with this scheme, the proposed FD relaying can reduce the total communication phases to one, thus has the potential to improve the throughput.

\section{B. Two-phase one-way FD}

Another scheme that we will compare with is the FD oneway relaying in which $R$ works in the FD mode while the two sources work in the HD mode. In this way, both sources can transmit with the maximum power. We use the same notation as the proposed scheme and the relay beamforming matrix is $\mathbf{W}=\mathbf{w}_{t} \mathbf{w}_{r}^{\dagger}$.

positive root within $\left(p_{B}^{\min }, p_{B}^{\max }\right)$ is denoted as $\Psi$ which may set $\Psi \cup\left\{p_{B}^{\min }, p_{B}^{\max }\right\}$ and choose the one that results in the maximum objective value.

to maximize the sum rate is dominated by the optimization of $\mathbf{w}_{t}$ and more specially, solving the problem (35). Since (35) is a semi-definite programming (SDP) problem with one variable $\mathbf{W}_{t} \in \mathbb{C}^{M_{t} \times M_{t}}$ and two constraints, the worst-case solution accuracy [29].

\section{BENCHMARK SCHEMES}

In this section, we introduce three benchmark schemes that the proposed FD network scheme can be compared with. The analog network coding, which is known to outperform the three-phase and four-phase HD schemes to provide throughput 35]; the second one is a two-phase one-way FD scheme last one ignores the residual SI channel at the relay thus provides a performance upper bound that is useful to evaluate

\section{A. Two-phase HD relaying using analog network coding}

HD analog network coding is introduced in [3] which takes $B$. In the firt phase, both sources transmit to $R$ and in the second phase, the relay multiplies the received signal by a mforming matrix $\mathbf{W}$ then broadcasts it to $\mathrm{A}$ and $\mathrm{B}$. Because needs to be optimized. The achievable rate pair and the relay power consumption, are given by

$$
\begin{aligned}
R_{A} & =\frac{1}{2} \log _{2}\left(1+\frac{P_{B}\left|\mathbf{h}_{R A}^{\dagger} \mathbf{W} \mathbf{h}_{B R}\right|^{2}}{\left\|\mathbf{h}_{R A}^{\dagger} \mathbf{W}\right\|^{2}+1}\right), \\
R_{B} & =\frac{1}{2} \log _{2}\left(1+\frac{P_{A}\left|\mathbf{h}_{R B}^{\dagger} \mathbf{W} \mathbf{h}_{A R}\right|^{2}}{\left\|\mathbf{h}_{R B}^{\dagger} \mathbf{W}\right\|^{2}+1}\right), \\
p_{R} & =\left\|\mathbf{W} \mathbf{h}_{A R}\right\|^{2} P_{A}+\left\|\mathbf{W} \mathbf{h}_{B R}\right\|^{2} P_{B}+\operatorname{trace}\left(\mathbf{W} \mathbf{W}^{\dagger}\right) .
\end{aligned}
$$

where the factor of $\frac{1}{2}$ is due to the two transmission phases used. Given the above rates and the power expression, prob-$$
\mathbf{W}=\mathbf{w}_{t} \mathbf{w}_{r}^{\dagger} \text {. }
$$ 
The achievable rate, relay power constraint, and zero residual SI constraint for source A (direction: B $\rightarrow$ A) are, respectively,

$$
\begin{aligned}
& R_{A}=\log _{2}\left(1+\frac{P_{B}\left|\mathbf{h}_{R A}^{\dagger} \mathbf{w}_{t}\right|^{2}\left|\mathbf{w}_{r}^{\dagger} \mathbf{h}_{B R}\right|^{2}}{\left|\mathbf{h}_{R A}^{\dagger} \mathbf{w}_{t}\right|^{2}+1}\right), \\
& p_{B}\left\|\mathbf{w}_{t}\right\|^{2}\left|\mathbf{w}_{r}^{\dagger} \mathbf{h}_{B R}\right|^{2}+\left\|\mathbf{w}_{t}\right\|^{2}\left\|\mathbf{w}_{r}\right\|^{2} \leq P_{R}, \\
& \mathbf{w}_{r}^{\dagger} \mathbf{H}_{R R} \mathbf{w}_{t}=0 .
\end{aligned}
$$

In our previous work [19], we have derived the closed-form expressions below for $R_{A}$ depending on how the $\mathrm{ZF}$ constraint is realized:

1) Receive ZF. In this case, we assume $\mathbf{w}_{t}=\mathbf{h}_{R A}$ and choose $\mathbf{w}_{r}$ to achieve (46). We showed that the achievable e2e received signal-to-noise ratio (SNR) can be expressed as

$$
\gamma_{R Z F}=\frac{P_{B}\left\|\mathbf{D h}_{B R}\right\|^{2} P_{R}\left\|\mathbf{h}_{R A}\right\|^{2}}{P_{B}\left\|\mathbf{D h}_{B R}\right\|^{2}+P_{R}\left\|\mathbf{h}_{R A}\right\|^{2}+1},
$$

where $\mathbf{D} \triangleq \Pi_{\mathbf{H}_{R R} \mathbf{h}_{R A}}^{\perp}$.

2) Transmit ZF. In this case, we assume $\mathbf{w}_{r}=\mathbf{h}_{B R}$ and choose $\mathbf{w}_{t}$ to achieve (46). We then reach the following achievable SNR:

$$
\gamma_{T Z F}=\frac{P_{B}\left\|\mathbf{h}_{B R}\right\|^{2} P_{R}\left\|\mathbf{B} \mathbf{h}_{R A}\right\|^{2}}{P_{B}\left\|\mathbf{h}_{B R}\right\|^{2}+P_{R}\left\|\mathbf{B} \mathbf{h}_{R S}\right\|^{2}+1},
$$

where $\mathbf{B} \triangleq \Pi_{\mathbf{H}_{R R}^{\dagger}}^{\perp} \mathbf{h}_{B R}$.

$R_{A}$ is then determined by $R_{A}=\log _{2}\left(1+\max \left(\gamma_{R Z F}, \gamma_{T Z F}\right)\right)$. We can derive similar achieve rate $R_{B}$ for the source $\mathrm{B}$.

Note that $R_{A}$ and $R_{B}$ cannot be achieved simultaneously as it requires that each corresponding source occupies the whole transmission time. The boundary of the rate region can be obtained by using time-sharing parameter $t \in[0,1]$, i.e., $\left(t R_{A},(1-t) R_{B}\right)$.

\section{FD-Upper Bound}

This scheme is the same as the proposed FD scheme except that we assume there is no SI at $\mathrm{R}$, but we still consider the SI at the two sources, i.e., $\mathbf{H}_{R R}=\mathbf{0},\left|h_{A A}\right|>0,\left|h_{B B}\right|>0$. In this case the ZF constraint in (13) is not necessary. We remark that this scheme uses unrealistic assumption of $\mathbf{H}_{R R}=\mathbf{0}$, so it is not a practical scheme but provides a useful upper bound to evaluate the performance of the proposed algorithms.

In the simulation results, we will label the above three benchmark schemes as "Two-phase HD", "Two-phase FD" and "Proposed one-phase FD upper bound", respectively.

\section{NUMERICAL RESUlts}

In this section we provide numerical results to illustrate the achievable rate region and the sum rate performance of the proposed FD two-way relaying scheme. We compare it with the above mentioned three benchmark schemes. The simulation set-up follows the system model in Section II. Unless otherwise specified, we assume that there are $M_{T}=$ $M_{R}=3$ antennas at $\mathrm{R}$, the average residual SI channel gain is $\sigma_{A}^{2}=\sigma_{B}^{2}=\sigma_{R}^{2}=-20 \mathrm{~dB}$, and the per-node transmit
SNR for both sources and the relay is $P_{A}=P_{B}=P_{R}=10$ $\mathrm{dB}$, which are the power constraints in problem formulations $\mathbb{P}_{1}$ and $\mathbb{P}_{2}$. The results are obtained using 100 independent channel realizations.

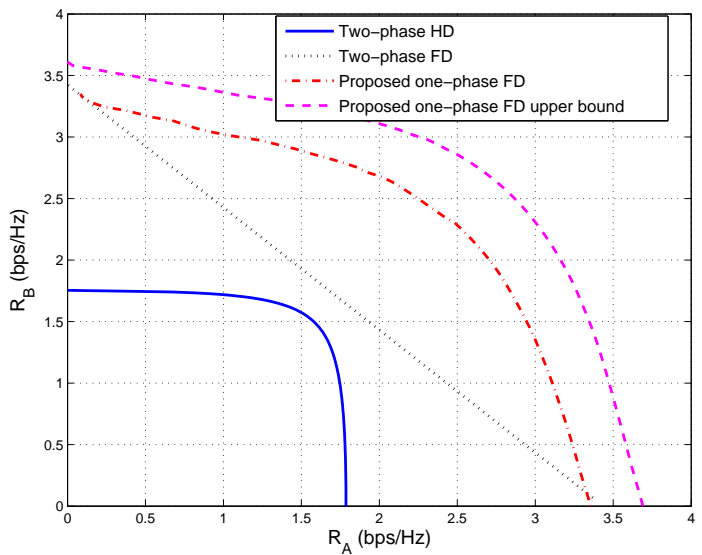

Fig. 2. Comparison of the achievable rate region. The residual SI channel gain is $-20 \mathrm{~dB}$. Transmit SNR is $10 \mathrm{~dB}$ at all nodes.

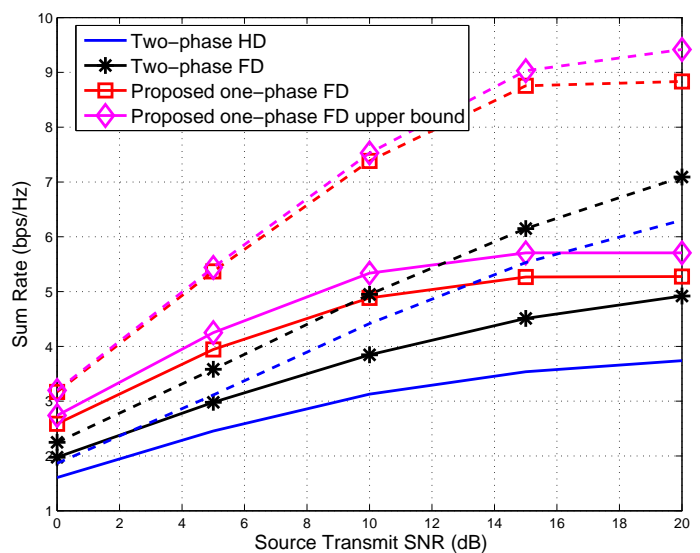

Fig. 3. Comparison of the sum rate. Solid curves are for $P_{R}=10 \mathrm{~dB}$ while dashed curves are for $P_{R}=20 \mathrm{~dB}$.

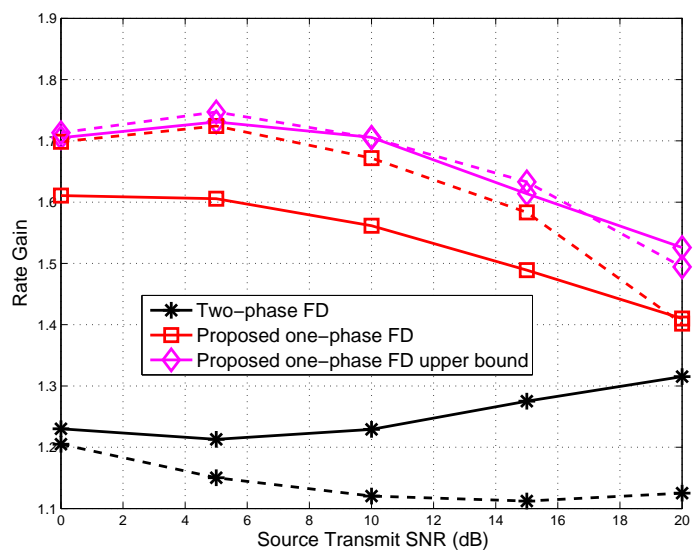

Fig. 4. Comparison of the sum rate gain over the conventional two-phase HD scheme. Solid curves are for $P_{R}=10 \mathrm{~dB}$ while dashed curves are for $P_{R}=20 \mathrm{~dB}$ 


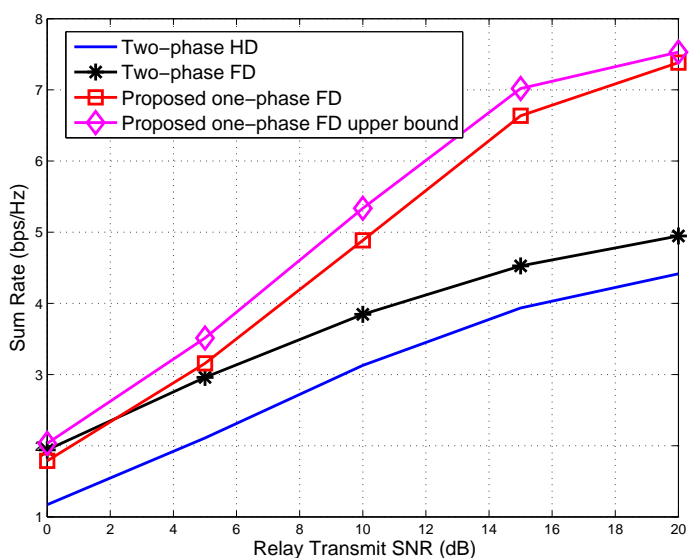

Fig. 5. The effect of the relay transmit SNR on the sum rate, $P_{A}=P_{B}=10$ dB.

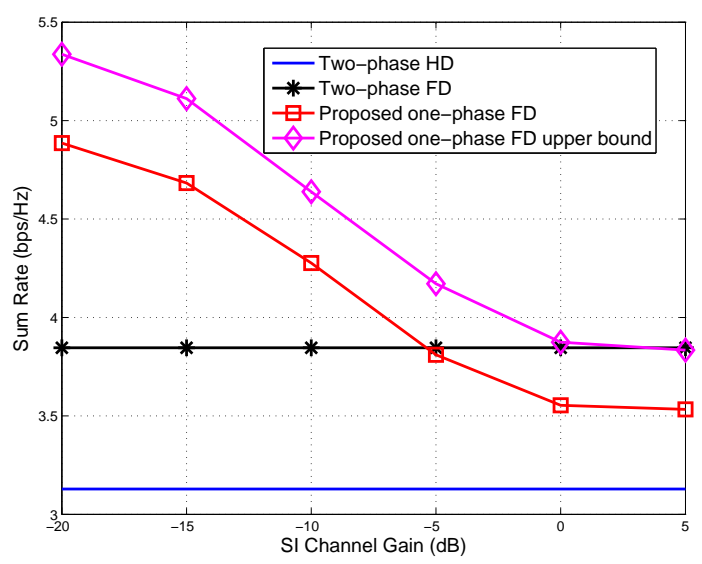

Fig. 6. The effect of the SI channel gain on the sum rate, $P_{A}=P_{B}=10$ dB.

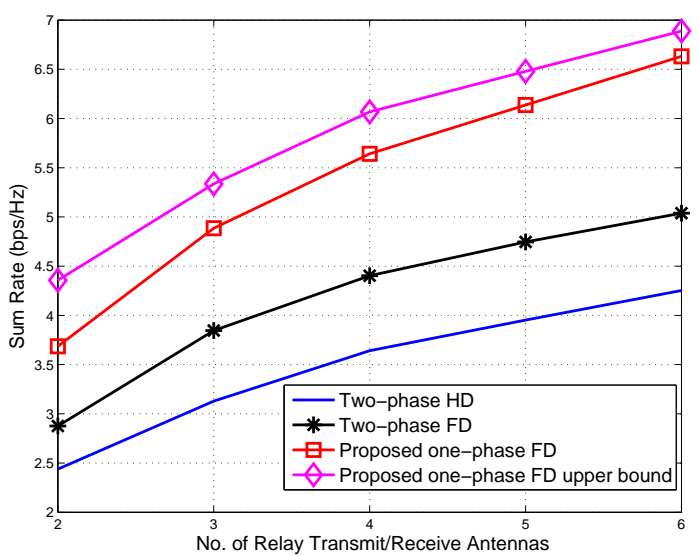

Fig. 7. The effect of the number of the relay antennas on the sum rate, $P_{A}=P_{B}=P_{R}=10 \mathrm{~dB}$.

\section{A. Achievable rate region}

First we illustrate the achievable average rate region for the two sources A and B in Fig. 2. It is seen that the two-phase FD scheme already greatly enlarges the achievable data region of the conventional HD scheme. The proposed one-phase FD scheme achieves significantly larger rate region over the twophase FD and the conventional HD schemes. We observe that

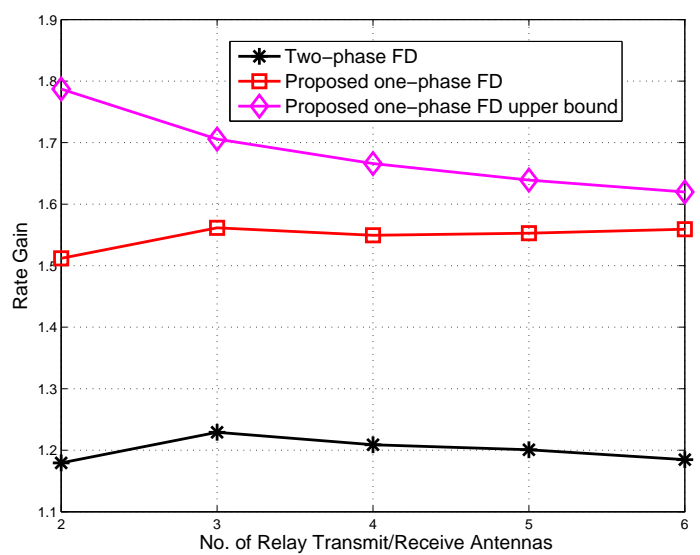

Fig. 8. Rate gain vs. no. of relay antennas, $P_{A}=P_{B}=P_{R}=10 \mathrm{~dB}$.

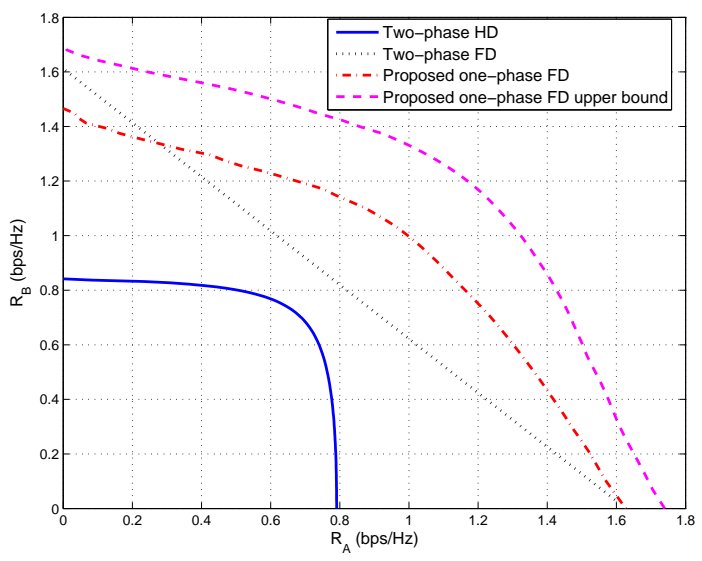

Fig. 9. Rate region for the asymmetric case, where the average channel gain between $R$ and $B$ is $-10 \mathrm{~dB}$.

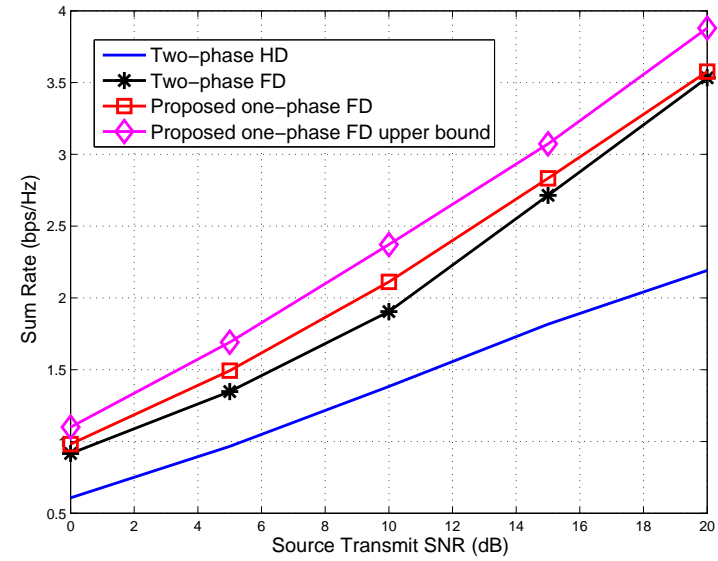

Fig. 10. Sum rate vs. source transmit SNR, $P_{R}=20 \mathrm{~dB}$ for the asymmetric case, where the average channel gain between $R$ and $B$ is $-10 \mathrm{~dB}$.

there is still a noticeable gap between the proposed scheme and its upper bound because the proposed solution is suboptimal.

\section{B. Sum rate performance}

We then investigate the effect of the source transmit SNR $P_{A}$ and $P_{B}\left(P_{A}=P_{B}\right)$ on the sum rate shown in Fig. 3 when $P_{R}=10 \mathrm{~dB}$ (solid curves) and $P_{R}=20 \mathrm{~dB}$ (dashed 


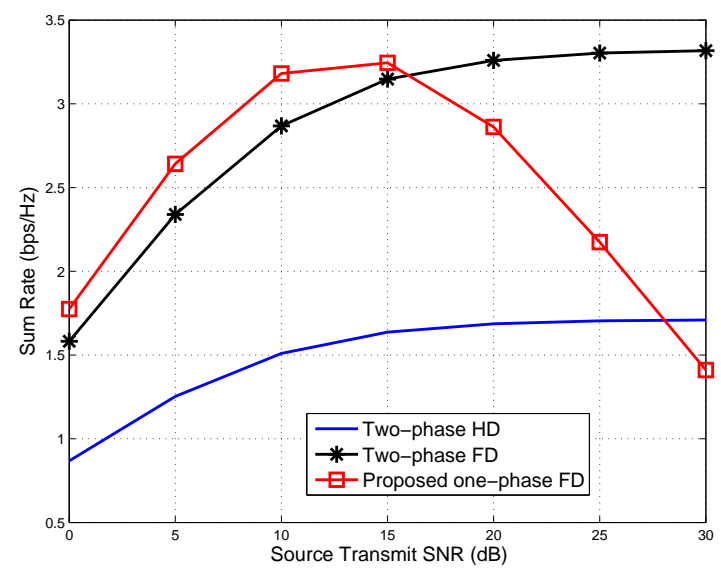

Fig. 11. Sum rate vs. source transmit SNR, $P_{R}=10 \mathrm{~dB}$ with only the receive CSI.

curves), respectively. We first consider the case $P_{R}=10 \mathrm{~dB}$. As expected, the sum rate improves as the source transmit SNR increase and the proposed one-phase FD schemes clearly outperforms the two benchmark schemes. When the source SNR is above $15 \mathrm{~dB}$, the sum rate of the proposed FD scheme saturates. This is because the high transmit power results in high residual SI, therefore increasing power budget does not necessarily improve the performance. To illustrate the performance improvement in sum rate, we show the sum rate gain over the conventional two-phase HD scheme in Fig. 4. It is observed that when the source SNR is $10 \mathrm{~dB}$, the proposed one-phase FD scheme and the two-phase FD scheme can achieve the sum rate gain of 1.56 and 1.22 , respectively. Even the performance upper bound cannot achieve double rate because of the residual SI at both sources. The rate gain in general decreases as the source transmit SNR increases again because of the residual SI therefore the sources need to carefully adjust its transmit power.

The same trend is observed when $P_{R}=20 \mathrm{~dB}$. In this case, a sum rate gain of nearly 1.7 is recorded when the source transmit SNR is $10 \mathrm{~dB}$. Another interesting observation is that the performance of the proposed scheme is very close to the upper bound. This is because when the relay power is high, the e2e performance is limited by the link from the source to the relay, rather than the relay to the other source, therefore the residual SI at the relay has little effect on the sum rate.

The impact of the relay transmit SNR on the sum rate is shown in Fig. 5. It is seen that when the relay SNR is low, the proposed one-phase FD scheme achieves lower sum rate than the two-phase FD scheme at low transmit SNRs then outperforms the latter when the transmit SNR is above $5 \mathrm{~dB}$. The performance gain is remarkable when the relay transmit SNR is high. This is because unlike the two sources, the relay can null out the residual SI using multiple antennas, therefore it can always use the maximum available power to improve the sum rate.

The effect of the residual SI channel gain at the sources is examined in Fig. 6. Naturally, the sum rate decreases as the residual SI channel becomes stronger or the SI is not adequately suppressed. When the residual SI channel gain is above $-5 \mathrm{~dB}$, the two-phase FD scheme outperforms the onephase FD scheme while both still achieve higher sum rate than the conventional two-phase HD scheme even when the SI channel gain is as high as $5 \mathrm{~dB}$.

Next we show the sum rate and rate gain results when the number of antennas $\left(M_{R}=M_{T}\right)$ at the relay varies from 2 to 6 in Fig. 7 and 8 respectively. The sum rate steady increases as more antennas are placed at the relay due to the array gain. It is observed that the rate gain remains about 1.55 when the number of antennas is greater than 2 .

\section{Asymmetric channel gain}

The above results are mainly for a symmetric case, i.e., both sources have similar power constraints and channel strengths. Here we consider an asymmetric case where the average channel gain between $R$ and $B$ is $-10 \mathrm{~dB}$. We plot the rate region in Fig. 9 using the same system parameters as those in Fig. 2 except that the gain of channel vectors $\mathbf{h}_{B R}$ and $\mathbf{h}_{R B}$ is $10 \mathrm{~dB}$ weaker. The results show that both sources' rates are reduced while the source $B$ suffers more rate loss. This is because one source's channels to and from the relay will also affect the performance of the other source. The sum rate comparison is given in Fig. 10 and it is observed the performance of the two-phase FD scheme is very close to that of the proposed FD scheme at all SNR region. This can be explained by the fact that e2e performance is restricted by the channel quality between $R$ and $B$, so the gain due to the simultaneous transmission of two sources is limited.

\section{Impact of the local channel state information}

Finally we consider the case that only the receive CSI at each node is available but the transmit CSI is unknown. Because of the lack of the transmit CSI, the two sources use full power $P_{A}$ and $P_{B} ; \mathbf{w}_{t}$ at the relay is chosen arbitrarily to satisfy the $\mathrm{ZF}$ constraint and the relay power constraint. The sum rate performance is shown in Fig. 11 It is seen that the proposed FD scheme still achieves significant performance gain over the HD relaying at low to medium transmit SNRs although all rates are much lower than the case with the global CSI in Fig. 3. Another notable difference is that at high transmit SNRs, the performance of the proposed FD scheme degrades quickly. This is because the two sources need to adjust its transmit power rather than using full power. This highlights the importance of the global CSI for adapting the transmit power and adjusting the relay beamforming.

\section{CONCLUSION AND FUTURE WORK}

We have investigated the application of the FD operation to MIMO TWRC, which requires only one phase for the two sources to exchange information. We studied two problems of finding the achievable rate region and maximizing the sum rate by optimizing the relay beamforming matrix and power allocation at the sources. Iterative algorithms are proposed together with 1-D search to find the local optimum solutions. At each iteration, either analytical solution or convex formulation has been derived. We have conducted intensive simulations to 
illustrate the effects of different system parameters. The results show that the FD operation has great potential to achieve much higher data rates than the conventional HD TWRC.

Regarding the future directions, better suboptimal and the optimal solutions are worth studying. There are a couple of reasons why the proposed algorithm is sub-optimal such as the additional $\mathrm{ZF}$ constraint, the incomplete characterization of the receive beamforming vector, and the alternating optimization algorithms. Another direction is to study the use of multiple transmit/receive antennas at the two sources. If a single data stream is transmitted, the residual SI can be removed using the $\mathrm{ZF}$ criterion at the sources as well. This actually simplifies the optimization as the two sources can use the maximum power. However, multiple antennas can support multiple and variable number of data streams, and when the problem is coupled with the SI suppression, it will be much more challenging. Thirdly, in this paper, we focus on the benefit of the FD in terms of spectrum efficiency. In [35], it is shown that for the HD case, three-phase transmission schemes offers a better compromise between the sum rate and the bit error rate than the twophase scheme, especially in the asymmetric case. It is worth investigating whether such an trade-off also exists for the FD scenario.

\section{REFERENCES}

[1] 3GPP TR 36.814 v.9.0.0 "Technical specification group radio access network; evolved universal terrestrial radio access (E-UTRA); Further advancements for E-UTRA physical layer aspects," March 2010.

[2] Z. Ding, I. Krikidis, B. Rong, J. S. Thompson, C. Wang, and S. Yang, "On combating the half-duplex constraint in modern cooperative networks: Protocols and techniques," IEEE Wireless Commun., vol. 19, no. 6, pp. 20-27, Dec. 2012.

[3] R. Zhang, Y. Liang, and C. C. Chai, "Optimal beamforming for twoway multi-antenna relay channel with analogue network coding," IEEE J. Select. Areas Commun., vol. 27, no. 5, pp. 699-712, June 2009.

[4] Q. Li, S. H. Ting, A. Pandharipande, and Y. Han, "Adaptive two-way relaying and outage analysis," IEEE Trans. Wireless Commun., vol. 8, no. 6, pp. 3288-3299, June 2009.

[5] D. W. Bliss, P. A. Parker, and A. R. Margetts, "Simultaneous transmission and reception for improved wireless network performance," in Proc. IEEE Workshop Statist. Signal Process., Madison, WI, Aug. 2007, pp. $478-482$

[6] D. W. Bliss, T. Hancock and P. Schniter, "Hardware and environmental phenomenological limits on full-duplex MIMO relay performance," in Proc. 46th Asilomar Conf. on Signals, Systems, and Computers, Pacific Grove, CA, Nov. 2012, pp. 1-6.

[7] T. Riihonen, S. Werner, R. Wichman, and E. B. Zacarias, "On the feasibility of full-duplex relaying in the presence of loop interference," in IEEE Int. Workshop on Signal Processing Advances in Wireless Communications (SPAWC) 2009, Perugia, Italy, June 2009, pp. 275-279.

[8] T. Riihonen, S. Werner, and R. Wichman, "Hybrid full-duplex/halfduplex relaying with transmit power adaptation," IEEE Trans. Wireless Commun., vol. 10, no. 9, pp. 3074-3085, Sept. 2011.

[9] B. P. Day, A. R. Margetts, D. W. Bliss, and P. Schniter, "Full-duplex MIMO relaying: achievable rates under limited dynamic range," IEEE J. Select. Areas Commun., vol. 30, no. 8, pp. 1541-1553, Sept. 2012.

[10] A. Sabharwal, P. Schniter, D. Guo, D. Bliss, S. Rangarajan, and R. Wichman, "In-band FD wireless: challenges and opportunities," IEEE J. Sel. Area Commun., vol. 32, no. 9, pp. 1637-1652, Oct. 2014.

[11] E. Everett, A. Sahai, and A. Sabharwal, "Passive self-interference suppression for FD infrastructure nodes," IEEE Trans. Wireless Commun., vol. 13, no. 2, pp. 680-694, Jan. 2014.

[12] M. Duarte, C. Dick, and A. Sabharwal, "Experiment-Driven Characterization of Full-Duplex Wireless Systems," IEEE Trans. Wireless Commun., vol. 11, no. 12, pp. 4296-4307, Dec. 2012.

[13] D. Bharadia, E. McMilin, and S. Katti, "Full duplex radios," in Proc. ACM Special Interest Group on Data Communication (SIGCOMM), Hong Kong, China, Aug. 2013, pp. 375-386.
[14] D. Bharadia, and S. Katti, "Full duplex MIMO radios," in 11th USENIX Symp. on Networked Systems Design and Implementation (NSDI 14), Seattle, WA, April, 2014.

[15] T. Riihonen, S. Werner, and R. Wichman, "Mitigation of loopback selfinterference in full-duplex MIMO relays," IEEE Trans. Signal Process., vol. 59, no.12, pp. 5983-5993, Dec. 2011.

[16] I. Krikidis, H. A. Suraweera, P. J. Smith and C. Yuen, "Full-duplex relay selection for amplify-and-forward cooperative networks," IEEE Trans. Wireless Commun., vol. 11, no. 12, pp. 4381-4393, Dec. 2012.

[17] D. Choi and D. Park, "Effective self interference cancellation in full duplex relay systems," Electron. Lett., vol. 48, no. 2, pp. 129-130, Jan. 2012.

[18] B. Chun and H. Park, "A spatial-domain joint-nulling method of self interference in full-duplex relays," IEEE Commun. Lett., vol. 16, no. 4 pp. 436-438, Apr. 2012.

[19] H. A. Suraweera, I. Krikidis, G. Zheng , C. Yuen, and P. J. Smith, "Low-complexity end-to-end performance optimization in mimo fullduplex relay systems," IEEE Trans. Wireless Commun., vol. 13, no. 2, pp. 913-927, Feb. 2014.

[20] B. Rankov and A. Wittneben, "Achievable rate regions for the two-way relay channel," in IEEE Int. Symp. Inf. Theory (ISIT), Seattle, WA, July 2006, pp. 1668-1672.

[21] W. Nam, S.-Y. Chung, and Y. H. Lee, "Capacity of the Gaussian twoway relay channel to within $1 / 2$ bit," IEEE Trans. Inf. Theory, vol. 56, no. 11, pp. 5488-5494, Nov. 2010.

[22] R. Hu, C. Hu, J. Jiang, X. Xie, and L. Song, "Full-duplex mode in amplify-and-forward relay channels: outage probability and ergodic capacity," International Journal of Antennas and Propagation, Volume 2014, Article ID 347540,8 pages.

[23] S. Tedik, G. K. Kurt, "Practical full duplex physical layer network coding," [online:] http://arxiv.org/abs/1310.6139

[24] H. Cui, M. Ma, L. Song, and B. Jiao, "Relay selection for two-way full duplex relay networks with amplify-and-forward protocol," IEEE Trans. Wireless Commun., to appear, 2014.

[25] X. Cheng, B. Yu, X. Cheng, L. Yang, "Two-way full-duplex amplifyand-forward relaying," in IEEE Military Communications Conference (MILCOM), San Diego, CA, Nov. 2013, pp. 1-6.

[26] K. Alexandris, A. Balatsoukas-Stimming, and A. Burg, "Measurementbased characterization of residual self-interference on a full-duplex MIMO testbed," IEEE Sensor Array and Multichannel Signal Processing Workshop, June 2014, A Coruna, Spain (to appear).

[27] M. Duarte, "Full-duplex wireless: Design, implementation and characterization," Ph.D. dissertation, Dept. Elect. and Computer Eng., Rice University, Houston, TX, 2012.

[28] E. A. Jorswieck and E. Larsson, "Complete Characterization of the Pareto Boundary for the MISO Interference Channel," IEEE Trans. on Signal Process., vol. 56, no. 10, pp. 5292-5296, Oct. 2008.

[29] Z.-Q. Luo, W.-K. Ma, A. M.-C. So, Y. Ye, and S. Zhang, "Semidefinite relaxation of quadratic optimization problems," IEEE Signal Process. Magazine, vol. 27, no. 3, pp. 20-34, May 2010.

[30] J. Li, A. P. Petropulu, and S. Weber, "On cooperative relaying schemes for wireless physical layer security," IEEE Trans. Signal Process., vol. 59, no. 10, pp. 4985-4997, Oct. 2011.

[31] S. Boyd and L. Vandenberghe, "Convex Optimization," Cambridge University Press, 2004.

[32] Le Thi Hoai An and Pham Dinh Tao, "The DC (difference of convex functions) programming and DCA revisited with DC models of real world nonconvex optimization problems," Annals of Operations Research, vol. 133, pp. 23-46, 2005.

[33] W. Ai, Y. Huang and S. Zhang, "New results on Hermitian matrix rankone decomposition," Mathematical Programming, Series A, vol. 128, No. 1-2, pp. 253-283, June 2011.

[34] A. Gjendemsj, D. Gesbert, G. E. Oien and S. G. Kiani "Binary power control for sum rate maximization over multiple interfering links," IEEE Trans. Wireless Commun., vol. 7, no. 8, pp. 3164-3173, Aug. 2008.

[35] Raymond H.Y. Louie, Y. Li, and B.Vucetic, "Practical physical layer network coding for two-way relay channels: performance analysis and comparison," IEEE Trans. Wireless Commun., vol. 9, no.2, pp. 764-777, Feb. 2010. 